\title{
Supporting information \\ Transcending Binary Logic by Gating Three Coupled Quantum Dots
}

Michael Klein, S. Rogge, F. Remacle and R. D. Levine

To determine the charge on dot $i$ we can solve the Heisenberg equations of motion for the expectation values of the operators $\hat{E}_{i, i}$ for the wave function that initially, at time $t \rightarrow-\infty$, is the ground state. Or we can propagate an initial state by converting the time dependent Schroedinger equation to a matrix differential equation for the expansion coefficients of the wave function in the zero order basis states, $i \hbar d \mathbf{c} / d t=\mathbf{H c}$. Here $\mathbf{c}(\mathrm{t})$ is a column vector of eight components such that $\mathbf{c}(t \rightarrow-\infty)$ are the expansion coefficients of the initial state of the array. In this letter the initial state is the ground state before the gating. $\mathbf{H}$ is the matrix representation of the Hamiltonian given in (1) in the zero order basis states. $\mathbf{H}$ is diagonal except for the matrix elements of the transfer operator, the term multiplied by $\beta$ in (1). See,

e.g., ${ }^{41}$ for explicit expressions for the matrix elements of the operators $\hat{E}$. In particular, note that the numbering of the states as shown in figure 2 facilitates the computation of the off diagonal matrix elements of $\hat{H}$. The occupancy of dot $i$ at the time $t$ is given by $\mathbf{c}^{\mathrm{T}} \hat{\mathbf{E}}_{i, i} \mathbf{c}$ where the superscript $\mathrm{T}$ denotes the transpose of the vector. When the time-dependent gating is over, the occupancy settles down to a constant value because the basis states are eigenstates of the site occupation operators $\hat{E}_{i, i}$. As a result of the gating, typically the long time state is such that the energy differences between the valence orbitals of the three dots are much larger than the exchange coupling, $\beta$. Then the zero-order basis states (see figure 2) are practically eigenstates of the Hamiltonian and of $\hat{E}_{i, i}$. To include the Coulomb repulsion the Hamiltonian has a bilinear term. Therefore propagating the time dependent Schroedinger equation requires taking a rather small time step. But a ns switching spans billions of such steps and care needs to be exercised that unitarity is not lost. It is the same dephasing that will cause problems for using the device as a quantum computer. For this reason we only read probabilities and do not rely on amplitudes. In our integration charge is conserved to better than $1 \%$.

For realistic weak coupling the switching of the gate potential on a ns scale results in an essentially adiabatic behavior so that, for practical purpose because the switching is far slower than the charge migration time, the system remains throughout in its (time-dependent) 
ground electronic state. It is therefore possible to simplify the computation by diagonalizing the Hamiltonian at every instant of time. If all one needs is to simulate the final state (= the function evaluation) then just one diagonalization, at a long time after the switching, is enough. We do not however take advantage of the adiabatic approximation except as an aid to our thinking. 